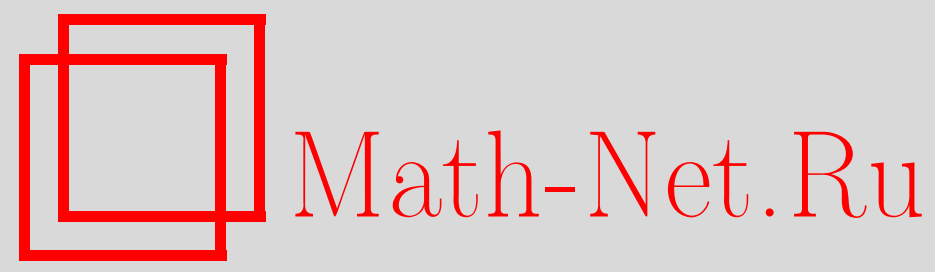

В. Г. Михайлов, А. М. Шойтов, Многократные повторения длинных цепочек в конечной цепи Маркова, Матем. вопр. криптогр., 2015, том 6, выпуск 3, 117-133

DOI: https://doi.org/10.4213/mvk163

Использование Общероссийского математического портала Math-Net.Ru подразумевает, что вы прочитали и согласны с пользовательским соглашением

http://www . mathnet.ru/rus/agreement

Параметры загрузки:

IP : 54.198 .187 .58

26 апреля 2023 г., 04:33:03

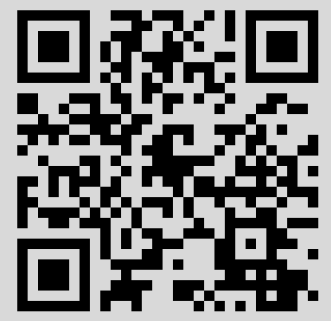


УДК: $519.212 .2+519.214$

\title{
Многократные повторения длинных цепочек в конечной цепи Маркова
}

\author{
В. Г. Михайлов ${ }^{1}$, А. М. Шойтов ${ }^{2}$ \\ ${ }^{1}$ Математический институт им. В. А. Стеклова РАН, Москва \\ ${ }^{2}$ Академия криптографии Российской Федерации, Москва
}

Получено 02.VI.2015

Пусть $X_{0}, X_{1}, \ldots$ - простая эргодическая цепь Маркова с конечным числом состояний и $\tilde{\xi}_{n, k}^{(m)}(s)-$ число $m$-серий $k$-кратных повторений $s$ цепочек в отрезке цепи $X_{0}, X_{1}, \ldots, X_{n+s+m}$. Получены достаточные условия сходимости к многомерному распределению Пуассона распределения вектора $\tilde{\Xi}_{n, k, M}(s)=\left(\tilde{\xi}_{n, k}^{(1)}(s), \ldots, \tilde{\xi}_{n, k}^{(M)}(s)\right)$ при $n, s \rightarrow \infty$, позволяющие доказывать предельные теоремы для некоторых связанных с $\tilde{\Xi}_{n, k, M}(s)$ случайных величин.

Ключевые слова: цепь Маркова, многократные повторения s-цепочек, многомерная предельная теорема Пуассона

\section{On multiple repetitions of long tuples in a Markov chain}

\section{G. Mikhailov ${ }^{1}$, A. M. Shoitov ${ }^{2}$}

${ }^{1}$ Steklov Mathematical Institute of RAS, Moscow

${ }^{2}$ Academy of Cryptography of the Russian Federation, Moscow

Abstract. Let $X_{0}, X_{1}, \ldots$ be a simple ergodic Markov chain with $N$ states and $\tilde{\xi}_{n, k}^{(m)}(s)$ be the number of $m$-series of $k$-repetitions of $s$-tuples in the chain segment $X_{0}, X_{1}, \ldots, X_{n+s+m}$. The sufficient conditions for the distribution of the vector $\tilde{\Xi}_{n, k, M}(s)=$ $=\left(\tilde{\xi}_{n, k}^{(1)}(s), \ldots, \tilde{\xi}_{n, k}^{(M)}(s)\right)$ to converge to the multidimensional Poisson distribution are found. This permits to prove limit theorems for the distributions of some random variables connected with $\tilde{\Xi}_{n, k, M}(s)$.

Key words: Markov chain, multiple repetitions of tuples, multidimensional Poisson limit theorem

Citation: Mathematical Aspects of Cryptography, 2015, vol. 6, no. 3, pp. 117-133 (Russian). 


\section{1. Введение}

Пусть последовательность $\mathbf{X}=\left\{X_{0}, X_{1}, \ldots\right\}$ образует простую неразложимую ациклическую однородную цепь Маркова с состояниями $1, \ldots, N$, матрицей переходных вероятностей $\mathbb{P}=\left\|p_{a, b}\right\|$ и произвольным начальным распределением. Отметим, что такая цепь является эргодической.

Положим $Y_{i}(s)=\left(X_{i}, \ldots, X_{i+s-1}\right), s, i=1,2, \ldots$, и для натурального $k, k \geq 2$, рассмотрим события (здесь и далее $\bar{E}$ обозначает дополнение к событию $E$ )

$$
\begin{aligned}
& E_{i_{1}, \ldots, i_{k}}=E_{i_{1}, \ldots, i_{k}}(s)=\left\{Y_{i_{1}}(s)=Y_{i_{2}}(s)=\ldots=Y_{i_{k}}(s)\right\}, \\
& E_{i_{1}, \ldots, i_{k}}^{(m)}=\bar{E}_{i_{1}-1, \ldots, i_{k}-1} \cap \bigcap_{j=0}^{m-1} E_{i_{1}+j, \ldots, i_{k}+j} \cap \bar{E}_{i_{1}+m, \ldots, i_{k}+m} .
\end{aligned}
$$

В [2] и [9] для события $E_{i_{1}, \ldots, i_{k}}$ был использован термин «k-кратное повторение цепочки». Событие $E_{i_{1}, \ldots, i_{k}}^{(m)}$ можно трактовать как серию из $m k$ кратных повторений $E_{i_{1}, \ldots, i_{k}}, \ldots, E_{i_{1}+m-1, \ldots, i_{k}+m-1}$. Очевидно, что

$$
\bigcap_{j=0}^{m-1} E_{i_{1}+j, \ldots, i_{k}+j}=E_{i_{1}, \ldots, i_{k}}(s+m-1) .
$$

Пусть

$$
\xi_{n, k}(s)=\sum_{1 \leq i_{1}<i_{2}<\ldots<i_{k} \leq n} I\left\{E_{i_{1}, \ldots, i_{k}}\right\}
$$

- число $k$-кратных повторений $s$-цепочек,

$$
\tilde{\xi}_{n, k}^{(m)}(s)=\sum_{1 \leq i_{1}<i_{2}<\ldots<i_{k} \leq n} I\left\{E_{i_{1}, \ldots, i_{k}}^{(m)}\right\}
$$

- число серий длины $m$, образованных $k$-кратными повторениями $s$-цепочек, начинающихся до момента $n$ включительно, а

$$
\tilde{\xi}_{n, k}(s)=\sum_{m \geq 1} \tilde{\xi}_{n, k}^{(m)}(s)
$$

- общее число серий, образованных $k$-кратными повторениями $s$-цепочек, начинающихся до момента $n$ включительно.

В работах [1-4] величины $Y_{i}(s), \xi_{n, k}(s)$ и $\tilde{\xi}_{n, k}(s)$ рассматривались в последовательности полиномиальных испытаний. В [2-4], в частности, было 
показано, что при $s, n \rightarrow \infty$ и $\mathbf{E} \tilde{\xi}_{n, k}(s) \rightarrow \lambda \in(0, \infty)$ распределения величин $\xi_{n, k}(s)$ и $\tilde{\xi}_{n, k}(s)$ сходятся к сложному пуассоновскому и пуассоновскому распределениям соответственно.

Задачи, связанные с совпадениями цепочек из двух независимых конечных цепей Маркова, рассматривались в [5]. Задачи о повторениях состояний и цепочек состояний в цепях Маркова в схемах с возрастающим числом исходов изучались в [6-8].

В [9] изучалось распределение величины $\tilde{\xi}_{n, 2}(s)$ в неразложимой ациклической марковской цепи. В частности, были выведены достаточные условия сходимости (при $n, s \rightarrow \infty$ ) распределения случайной величины $\tilde{\xi}_{n, 2}(s)$ к распределению Пуассона и к нормальному распределению (распределение цепи $\mathbf{X}$ считалось фиксированным). В качестве следствий этих предельных теорем были получены предельные теоремы для времени первого повторения $s$-цепочек $\tau(s)$ и для максимальной длины повторившейся цепочки $\mu(n)$.

В настоящей работе рассматривается случай повторений произвольной кратности. Получены достаточные условия сходимости распределения случайного вектора $\left(\tilde{\xi}_{n, k}^{(1)}(s), \ldots, \tilde{\xi}_{n, k}^{(M)}(s)\right)$ (при произвольном натуральном $M$ ) в неразложимой ациклической марковской цепи к многомерному распределению Пуассона. Эти же условия являются достаточными для сходимости распределения случайной величины $\tilde{\xi}_{n, k}(s)$ к распределению Пуассона и для сходимости распределения случайной величины $\xi_{n, k}(s)$ к сложному пуассоновскому распределению.

\section{2. Формулировка основного результата}

$$
\text { Пусть } p=\max _{a, b \in\{1, \ldots, N\}} p_{a, b}, \pi=\left(\pi_{1}, \ldots, \pi_{N}\right), \pi_{r}>0, r=1, \ldots, N,-
$$
стационарное распределение цепи $\mathbf{X}, \Pi_{k}=\sum_{r=1}^{N} \pi_{r}^{k}$. Рассмотрим матрицу $\mathbb{P}_{k}=$ $=\left\|p_{a, b}^{k}\right\|$. Матрица $\mathbb{P}_{k}$ является неразложимой и примитивной (см. [10]), так как расположение нулевых элементов в ней совпадает с расположением нулевых элементов в матрице $\mathbb{P}$. Пусть $\rho_{k}=\rho\left(\mathbb{P}_{k}\right)>0$ - максимальное характеристическое число матрицы $\mathbb{P}_{k}$, а $\left(z_{k, 1}, \ldots, z_{k, N}\right), z_{k, r}>0, r=1, \ldots, N$, собственный вектор, соответствующий характеристическому числу $\rho_{k}$.

Согласно предложению $2[10$, с. 365$]$ для $\mathbb{P}_{k}$ имеет место представление

$$
\mathbb{P}_{k}=Z_{k} \rho_{k} \mathbb{P}_{k}^{\prime} Z_{k}^{-1}
$$

где $Z_{k}$ - диагональная матрица, на главной диагонали которой расположены числа $z_{k, 1}, \ldots, z_{k, N}$, a $\mathbb{P}_{k}^{\prime}-$ стохастическая матрица. Матрица $\mathbb{P}_{k}^{\prime}$ определяет 
неразложимую и ациклическую цепь Маркова со стационарным распределением $\pi_{k}^{\prime}=\left(\pi_{k, 1}^{\prime}, \ldots, \pi_{k, N}^{\prime}\right), \pi_{k, r}^{\prime}>0, r=1, \ldots, N$, и для некоторой константы $c_{1}=c_{k, 1}>0$ при $s \rightarrow \infty$ справедлива оценка

$$
\left|\mathbb{P}_{k}^{\prime s}-\Pi_{k}^{\prime}\right|=O\left(e^{-c_{1} s}\right),
$$

где матрица $\boldsymbol{\Pi}_{k}^{\prime}$ состоит из одинаковых строк $\pi_{k}^{\prime}$, а $|A|$ - норма матрицы $A=\left\|a_{i, j}\right\|$, определяемая формулой (см. [10])

$$
|A|=\max _{1 \leq i \leq N} \sum_{j=1}^{N}\left|a_{i, j}\right| .
$$

Введем обозначения

$$
\begin{gathered}
\left(\pi_{k}^{\prime}, z_{k}^{-1}\right)=\sum_{r=1}^{N} \pi_{k, r}^{\prime} z_{k, r}^{-1}, \quad\left(\pi^{k}, z_{k}\right)=\sum_{r=1}^{N} \pi_{r}^{k} z_{k, r}, \\
\Lambda_{s, n}(k)=C_{n}^{k} \rho_{k}^{s-1}\left(1-\rho_{k}\right)\left(\pi_{k}^{\prime}, z_{k}^{-1}\right)\left(\pi^{k}, z_{k}\right) .
\end{gathered}
$$

Теорема 1. Пусть $n, s \rightarrow \infty$ и для фиксированного натурального $k \geq 2$ выполнены условия $p^{k}<\rho_{k}, \Lambda_{s, n}(k) \rightarrow \lambda \in(0, \infty)$. Тогда:

1) при любом натуральном $M$ распределение случайного вектора $\left(\tilde{\xi}_{n, k}^{(1)}(s), \ldots, \tilde{\xi}_{n, k}^{(M)}(s)\right)$ сходится к М-мерному пуассоновскому распределению с производящей функиией

$$
\varphi_{M}\left(z_{1}, \ldots, z_{M}\right)=\exp \left\{\sum_{m=1}^{M} \lambda \rho_{k}^{m-1}\left(1-\rho_{k}\right)\left(z_{m}-1\right)\right\},
$$

2) распределение случайной величины $\tilde{\xi}_{n, k}(s)$ сходится к распределению Пуассона с параметром $\lambda$,

3) распределение случайной величины $\xi_{n, k}(s)$ сходится к сложному пуассоновскому распределению с производящей функиией

$$
\varphi(z)=\exp \left\{\frac{\lambda(z-1)}{1-\rho_{k} z}\right\} .
$$

Замечание 1. Пусть строки матрицы $\mathbb{P}$ являются перестановками одного и того же фиксированного вектора $\left(p_{1}, \ldots, p_{N}\right), P_{k}=\sum_{i=1}^{N} p_{i}^{k}$. Тогда $\rho_{k}=P_{k}>p^{k}$, вектор $\left(z_{k, 1}, \ldots, z_{k, N}\right)=(1, \ldots, 1)$, а $\Lambda_{s, n}=C_{n}^{k} P_{k}^{s-1}\left(1-P_{k}\right) \Pi_{k}$. 
Замечание 2. В [9] приведены и другие примеры марковских цепей, для которых $p^{k}<\rho_{k}$. В общем случае вопрос выполнения этого условия требует отдельного изучения.

Замечание 3. Из условия $p^{k}<\rho_{k}$ теоремы 1 по свойствам спектрального радиуса (см. [10]) при $r \geq 2$ вытекают оценки

$$
\rho_{r}=\rho\left(\mathbb{P}_{r}\right) \leq \rho\left(p \mathbb{P}_{r-1}\right)=p \rho\left(\mathbb{P}_{r-1}\right)<\rho_{k}^{1 / k} \rho_{r-1},
$$

и, следовательно, $\rho_{k} \leq p^{k-1}$ и

$$
p<\rho_{k}^{1 / k}<\rho_{k-1}^{1 /(k-1)}<\ldots<\rho_{2}^{1 / 2}<1 .
$$

\section{3. Схема доказательства теоремы 1}

Введем обозначения

$$
\begin{gathered}
J=\left\{\bar{i}=\left(i_{1}, \ldots, i_{k}\right): 1 \leq i_{1}<\ldots<i_{k} \leq n\right\} \\
\eta(\bar{i}, s)=I\left\{Y_{i_{1}}(s)=Y_{i_{2}}(s)=\ldots=Y_{i_{k}}(s)\right\}, \quad \eta^{(m)}(\bar{i})=I\left\{E_{i_{1}, \ldots, i_{k}}^{(m)}\right\} .
\end{gathered}
$$

Для любого $\bar{i}$ из $J$ и натурального $h$ определим множество

$$
U^{(h)}(\bar{i})=\bigcup_{t=1}^{k}\left[i_{t}, i_{t}+h-1\right] .
$$

Для всех $r=1, \ldots, k$ положим

$J^{(h)}(r)=\left\{\bar{i} \in J\right.$, множество $U^{(h)}(\bar{i})$ состоит ровно из $r$ связных компонент $\}$.

Очевидно, что $J=\bigcup_{r=1}^{k} J^{(h)}(r)$ для любого $h$, и, следовательно,

$$
\xi_{n, k}(s)=\sum_{r=1}^{k} \sum_{\bar{i} \in J^{(h)}(r)} \eta(\bar{i}), \quad \tilde{\xi}_{n, k}^{(m)}(s)=\sum_{r=1}^{k} \sum_{\bar{i} \in J^{(h)}(r)} \eta^{(m)}(\bar{i}) .
$$

Возьмем $h=s^{3}$ и определим случайные величины

$$
\delta_{n, k}^{(m)}(s)=\sum_{\bar{i} \in J^{\left(s^{3}\right)}(k)} \eta^{(m)}(\bar{i}) .
$$


Отметим сразу, что

$$
\eta^{(m)}(\bar{i}) \leq \eta(\bar{i}, s+m-1), \quad \delta_{n, k}^{(m)}(s) \leq \tilde{\xi}_{n, k}^{(m)}(s) \leq \xi_{n, k}(s+m-1) .
$$

Введем обозначения

$$
\tilde{\Xi}_{n, k, M}(s)=\left(\tilde{\xi}_{n, k}^{(1)}(s), \ldots, \tilde{\xi}_{n, k}^{(M)}(s)\right), \quad \Delta_{n, k, M}(s)=\left(\delta_{n, k}^{(1)}(s), \ldots, \delta_{n, k}^{(M)}(s)\right) .
$$

Обозначим через $\mathcal{L}(W)$ распределение случайной величины (или вектора) $W$, через $\mathrm{Po}(\lambda)$ - распределение Пуассона с параметром $\lambda$, а через $\mathrm{Po}\left(\lambda_{1}, \ldots, \lambda_{M}\right)-M$-мерное распределение Пуассона с производящей функцией

$$
\varphi_{\lambda_{1}, \ldots, \lambda_{M}}\left(z_{1}, \ldots, z_{M}\right)=\exp \left\{\sum_{m=1}^{M} \lambda_{m}\left(z_{m}-1\right)\right\} .
$$

Пусть $d(\Phi, \Psi)$ - расстояние по вариации между распределениями $\Phi$ и $\Psi$.

Доказательство первого утверждения теоремы 1 состоит из трех шагов. Сначала доказывается соотношение

$$
\mathbf{P}\left\{\tilde{\Xi}_{n, k, M}(s) \neq \Delta_{n, k, M}(s)\right\} \rightarrow 0,
$$

затем - соотношение

$$
d\left(\mathcal{L}\left(\Delta_{n, k, M}(s)\right), \operatorname{Po}\left(\left(\mathbf{E} \delta_{n, k}^{(1)}(s), \ldots, \mathbf{E} \delta_{n, k}^{(M)}(s)\right)\right)\right) \rightarrow 0 .
$$

Кроме того, доказывается соотношение

$$
\operatorname{Po}\left(\left(\mathbf{E} \delta_{n, k}^{(1)}(s), \ldots, \mathbf{E} \delta_{n, k}^{(M)}(s)\right)\right) \rightarrow \operatorname{Po}\left(1-\rho_{k}, \rho_{k}\left(1-\rho_{k}\right), \ldots, \rho_{k}^{M-1}\left(1-\rho_{k}\right)\right) .
$$

Из (8)-(10) вытекает первое утверждение теоремы 1.

Утверждения 2) и 3) теоремы 1 доказываются по аналогичной схеме.

\section{4. Доказательство соотношения (8)}

Используя неравенство $\mathbf{P}\left\{X_{j}=b \mid X_{i}=a\right\} \leq p, j>i$, получаем для неперекрывающихся цепочек длины $s\left(i_{r} \geq i_{r-1}+s, r=2, \ldots, k\right)$ оценки

$$
\begin{gathered}
\mathbf{P}\left\{E_{i_{1}, \ldots, i_{k}}\right\}=\mathbf{P}\left\{Y_{i_{1}}(s)=Y_{i_{2}}(s)=\ldots=Y_{i_{k}}(s)\right\}= \\
=\sum_{\left(x_{1}, \ldots, x_{s}\right) \in\{1, \ldots, N\}^{s}} \mathbf{P}\left\{X_{i_{1}}=x_{1}\right\} \mathbf{P}\left\{X_{i_{2}}=x_{1} \mid X_{i_{1}+s-1}=x_{s}\right\} \prod_{r=2}^{s} p_{x_{r-1}, x_{r}} \times
\end{gathered}
$$




$$
\begin{aligned}
& \times \mathbf{P}\left\{X_{i_{3}}=x_{1} \mid X_{i_{2}+s-1}=x_{s}\right\} \prod_{r=2}^{s} p_{x_{r-1}, x_{r}} \cdot \ldots \cdot \mathbf{P}\left\{X_{i_{k}}=x_{1} \mid X_{i_{k-1}+s-1}=x_{s}\right\} \times \\
\times & \prod_{r=2}^{s} p_{x_{r-1}, x_{r}} \leq \sum_{\left(x_{1}, \ldots, x_{s}\right) \in\{1, \ldots, N\}^{s}} \mathbf{P}\left\{X_{i_{1}}=x_{1}\right\} p^{s(k-1)} p_{x_{1}, x_{2}} p_{x_{2}, x_{3}} \cdot \ldots \cdot p_{x_{s-1}, x_{s}}=p^{s(k-1)} .
\end{aligned}
$$

Значит, для неперекрывающихся цепочек длины $s+m-1$, т. е. при $i_{r} \geq i_{r-1}+$ $+s+M-1, r=2, \ldots, k, m \geq 1$

$$
\mathbf{P}\left\{Y_{i_{1}}(s+m-1)=Y_{i_{2}}(s+m-1)=\ldots=Y_{i_{k}}(s+m-1)\right\} \leq p^{(s+m-1)(k-1)} .
$$

Рассмотрим сначала случай перекрывающихся цепочек длины $s$. Пусть для определенности перекрываются цепочки $Y_{i_{1}}(s)$ и $Y_{i_{2}}(s)$ (возможно, не только они). Возьмем из всех других компонент связности множества $U^{(s)}(\bar{i})$ по одной представляющей их цепочке: $Y_{i_{2}}(s), \ldots, Y_{i_{j r}}(s)$. По аналогии с [2] и [11] заметим, что при $i_{2}=i_{1}+1, \ldots, i_{1}+s-1$ фиксация вектора $Y_{i_{1}}\left(i_{2}-i_{1}\right)=\left(x_{1}, \ldots, x_{i_{2}-i_{1}}\right)$ однозначно определяет все компоненты вектора $Y_{i_{2}}(s)=\left(x_{1}, \ldots, x_{i_{2}-i_{1}}, x_{1}, \ldots\right)$ и векторов $Y_{i_{2}}(s), \ldots, Y_{i_{k}}(s)$. Поэтому

$$
\begin{gathered}
\mathbf{P}\left\{Y_{i_{1}}(s)=Y_{i_{2}}(s)=\ldots=Y_{i_{k}}(s)\right\} \leq \\
\leq \mathbf{P}\left\{Y_{i_{1}}(s)=Y_{i_{2}}(s)=Y_{i_{j_{2}}}(s)=\ldots=Y_{i_{j_{r}}}(s)\right\} \leq p^{s r} .
\end{gathered}
$$

Заменив здесь $s$ на $s+m-1$, получим

$$
\mathbf{P}\left\{Y_{i_{1}}(s+m-1)=Y_{i_{2}}(s+m-1)=\ldots=Y_{i_{k}}(s+m-1)\right\} \leq p^{(s+m-1) r} .
$$

Теперь, используя неравенства (6), получаем

$$
\begin{gathered}
\mathbf{P}\left\{\tilde{\xi}_{n, k}^{(m)}(s) \neq \delta_{n, k}^{(m)}(s)\right\} \leq \mathbf{E}\left(\tilde{\xi}_{n, k}^{(m)}(s)-\delta_{n, k}^{(m)}(s)\right)= \\
=\sum_{r=1}^{k-1} \sum_{\bar{i} \in J^{(s+m-1)}(r)} \mathbf{E} \eta(\bar{i}, s+m-1)+\sum_{\bar{i} \in J^{(s+m-1)}(k) \backslash J^{\left(s^{3}\right)}(k)} \mathbf{E} \eta(\bar{i}, s+m-1) \leq \\
\leq \sum_{r=1}^{k-1}\left|J^{(s+m-1)}(r)\right| p^{(s+m-1) r}+\left|J^{(s+m-1)}(k) \backslash J^{\left(s^{3}\right)}(k)\right| p^{(s+m-1)(k-1)} \leq \\
\leq \sum_{r=1}^{k-1} n^{r}\left|J^{(s+m-1)}(r)\right| p^{(s+m-1) r}+\left|J^{(s+m-1)}(k) \backslash J^{\left(s^{3}\right)}(k)\right| p^{(s+m-1)(k-1)}=
\end{gathered}
$$




$$
=(s+m-1)^{k-1} \sum_{r=1}^{k-1} O\left(n^{r} p^{r(s+m-1)}\right)+(s+m-1)^{3(k-1)} O\left(n^{k-1} p^{(s+m-1)(k-1)}\right)
$$

равномерно по $m \geq 1$.

С учетом условия $p<\rho_{k}^{1 / k}$ (см. замечание 3 выше) получаем из (13), что равномерно по $m \geq 1$

$$
\mathbf{P}\left\{\tilde{\xi}_{n, k}^{(m)}(s) \neq \delta_{n, k}^{(m)}(s)\right\}=O\left((s+m-1)^{3 k}\left(\frac{p}{\rho_{k}^{1 / k}}\right)^{s+m-1}\right) .
$$

Суммируя эти оценки по $1 \leq m \leq M$, получаем

$$
\mathbf{P}\left\{\tilde{\Xi}_{n, k, M}(s) \neq \Delta_{n, k, M}(s)\right\}=O\left(s^{3 k}\left(\frac{p}{\rho_{k}^{1 / k}}\right)^{s}\right) \rightarrow 0 .
$$

Здесь использовано то, что функция $x^{3 k}\left(p \rho_{k}^{-1 / k}\right)^{x}$ стремится к нулю, когда $x$ стремится к бесконечности, и что при $s, n \rightarrow \infty$ выполнена оценка $s=$ $=O(\ln n)$, вытекающая из определения величин $\Lambda_{s, n}(k)$ и условий теоремы 1 . Соотношение (8) доказано.

\section{5. Доказательство соотношения (10)}

$$
\begin{aligned}
& \text { Оценим } \mathbf{E} \delta_{n, k}^{(m)}(s) \text {. Для } \bar{i} \in J^{\left(s^{3}\right)}(k) \text { имеем } \\
& \mathbf{P}\left\{E_{i_{1}, \ldots, i_{k}}\right\}=\mathbf{P}\left\{Y_{i_{1}}(s)=Y_{i_{2}}(s)=\ldots=Y_{i_{k}}(s)\right\}= \\
& =\sum_{\left(x_{1}, x_{2}, \ldots, x_{s}\right) \in\{1, \ldots, N\}^{s}} \mathbf{P}\left\{X_{i_{1}}=x_{1}\right\} \cdot p_{x_{s}, x_{1}}^{\left(i_{2}-i_{1}-s-1\right)} \cdot p_{x_{s}, x_{1}}^{\left(i_{3}-i_{2}-s-1\right)} \cdot \ldots \cdot p_{x_{s}, x_{1}}^{\left(i_{k}-i_{k-1}-s-1\right)} \times \\
& \quad \times p_{x_{1}, x_{2}}^{k} \cdot p_{x_{2}, x_{3}}^{k} \cdot \ldots \cdot p_{x_{s-1}, x_{s}}^{k} .
\end{aligned}
$$

Так как для $\bar{i} \in J^{\left(s^{3}\right)}(k)$ справедливы оценки $i_{r}-i_{r-1}>s^{3}, r=2, \ldots, k$, $i_{1}>s^{3}$, а цепь Маркова $\mathbf{X}$ является эргодической, то для некоторой константы $c_{2}>0$ равномерно по $\bar{i} \in J^{\left(s^{3}\right)}(k)$ и $x_{1}, x_{s} \in\{1, \ldots, N\}$ справедливы оценки

$$
\left|p_{x_{s}, x_{1}}^{\left(i_{r}-i_{r-1}-s-1\right)}-\pi_{x_{1}}\right|<e^{-c_{2} s^{3}}, \quad\left|\mathbf{P}\left\{X_{i_{1}}=x_{1}\right\}-\pi_{x_{1}}\right|<e^{-c_{2} s^{3}} .
$$


Поэтому равномерно по $\bar{i} \in J^{\left(s^{3}\right)}(k)$

$$
\begin{gathered}
\mathbf{P}\left\{Y_{i_{1}}(s)=Y_{i_{2}}(s)=\ldots=Y_{i_{k}}(s)\right\}= \\
=\sum_{\left(x_{1}, x_{2}, \ldots, x_{s}\right) \in\{1, \ldots, N\}^{s}} \pi_{x_{1}}^{k} \cdot p_{x_{1}, x_{2}}^{k} \cdot p_{x_{2}, x_{3}}^{k} \cdot \ldots \cdot p_{x_{s-1}, x_{s}}^{k}\left(1+O\left(e^{-c_{2} s^{3}}\right)\right) .
\end{gathered}
$$

По аналогии с (15), используя также (1) и соотношение $e^{-c_{2} s^{3}}=$ $=O\left(e^{-c_{1} s}\right)$ при $s \rightarrow \infty$, нетрудно получить записанное в векторном виде равенство

$$
\begin{gathered}
\left(\mathbf{P}\left\{Y_{i_{1}}(s)=Y_{i_{2}}(s)=\ldots=Y_{i_{k}}(s), X_{i_{1}+s-1}=a\right\}, a=1, \ldots, N\right)= \\
=\rho_{k}^{s-1}\left(1+O\left(e^{-c_{1} s}\right)\right)\left(\pi_{k, r}^{\prime} z_{k, r}^{-1} \sum_{l=1}^{N} \pi_{l}^{k} z_{k, l}, r=1, \ldots, N\right) .
\end{gathered}
$$

Тогда (сразу заменяем $s$ на $s+m-1$ )

$$
\begin{gathered}
\mathbf{P}\left\{Y_{i_{1}}(s+m-1)=Y_{i_{2}}(s+m-1)=\ldots=Y_{i_{k}}(s+m-1)\right\}= \\
=\rho_{k}^{s+m-2} \sum_{r=1}^{N} \pi_{k, r}^{\prime} z_{k, r}^{-1} \sum_{l=1}^{N} \pi_{l}^{k} z_{k, l} \cdot\left(1+O\left(e^{-c_{1}(s+m-1)}\right)\right) .
\end{gathered}
$$

Отсюда и из равенства

$$
\begin{aligned}
& \mathbf{P}\left\{E_{i_{1}, \ldots, i_{k}}^{(m)}\right\}=\mathbf{P}\left\{\bar{E}_{i_{1}-1, \ldots, i_{k}-1} \cap \bigcap_{j=0}^{m-1} E_{i_{1}+j, \ldots, i_{k}+j} \cap \bar{E}_{i_{1}+m, \ldots, i_{k}+m}\right\}= \\
&=\mathbf{P}\left\{\bigcap_{j=0}^{m-1} E_{i_{1}+j, \ldots, i_{k}+j}\right\}-\mathbf{P}\left\{\bigcap_{j=-1}^{m-1} E_{i_{1}+j, \ldots, i_{k}+j}\right\}- \\
&-\mathbf{P}\left\{\bigcap_{j=0}^{m} E_{i_{1}+j, \ldots, i_{k}+j} \cap E_{i_{1}+m, \ldots, i_{k}+m}\right\}+\mathbf{P}\left\{\bigcap_{j=-1}^{m} E_{i_{1}+j, \ldots, i_{k}+j}\right\}
\end{aligned}
$$

получаем (используя обозначения (3)), что равномерно по $\bar{i} \in J^{\left(s^{3}\right)}(k)$ и по $m \geq 1$

$$
\mathbf{P}\left\{E_{i_{1}, \ldots, i_{k}}^{(m)}\right\}=\rho_{k}^{s+m-2}\left(1-\rho_{k}\right)^{2}\left(\pi_{k}^{\prime}, z_{k}^{-1}\right)\left(\pi^{k}, z_{k}\right)\left(1+O\left(e^{-c_{1} s}\right)\right) .
$$


Следовательно (опять см. (3)), равномерно по $m \geq 1$

$$
\mathbf{E} \delta_{n, k}^{(m)}(s)=\Lambda_{s, n}(k) \rho_{k}^{m-1}\left(1-\rho_{k}\right)\left(1+O\left(\frac{s^{3}}{n}+e^{-c_{1} s}\right)\right) .
$$

Из соотношения (16) и условий теоремы следует, что

$$
\mathbf{E} \delta_{n, k}^{(m)}(s) \rightarrow \lambda \rho_{k}^{m-1}\left(1-\rho_{k}\right)
$$

равномерно по $m \geq 1$. Поэтому

$$
\varphi_{\mathbf{E} \delta_{n, k}^{(1)}(s), \ldots, \mathbf{E} \delta_{n, k}^{(M)}(s)}\left(z_{1}, \ldots, z_{M}\right) \rightarrow \varphi_{M}\left(z_{1}, \ldots, z_{M}\right),
$$

а значит, выполнено (10).

\section{6. Доказательство соотношения (9)}

Рассмотрим множество

$$
U=\left\{\alpha=(\bar{i}, m): \bar{i} \in J^{\left(s^{3}\right)}(k), m=1, \ldots, M\right\}
$$

и наборы случайных величин

$$
W=\left\{\eta^{(m)}(\bar{i})\right\}_{(\bar{i}, m) \in U}, \quad \Pi=\left\{\pi_{\bar{i}, m}\right\}_{(\bar{i}, m) \in U},
$$

где случайные величины $\pi_{\bar{i}, m}$, образующие набор П, независимы в совокупности и имеют распределения Пуассона с параметрами $\mathbf{E} \eta^{(m)}(\bar{i})=\mathbf{P}\left\{E_{i_{1}, \ldots, i_{k}}^{(m)}\right\}$ соответственно.

Заметим, что вектор $\Delta_{n, k, M}(s)$ может быть записан как результат применения к набору $W$ некоторого отображения $\Psi:\{0,1\}^{|U|} \rightarrow$ $\rightarrow\{0,1, \ldots\}^{M}$, а вектор $\Psi(\Pi)$ (с тем же самым $\Psi$ ) имеет распределение $\operatorname{Po}\left(\left(\mathbf{E} \delta_{n, k}^{(1)}(s), \ldots, \mathbf{E} \delta_{n, k}^{(M)}(s)\right)\right)$. Поэтому

$$
d\left(\mathcal{L}\left(\Delta_{n, k, M}(s)\right), \operatorname{Po}\left(\left(\mathbf{E} \delta_{n, k}^{(1)}(s), \ldots, \mathbf{E} \delta_{n, k}^{(M)}(s)\right)\right)\right) \leq d(W, \Pi),
$$

и для проверки (9) достаточно доказать следующее утверждение. 
Теорема 2. Пусть выполнены условия теоремы 1 . Тогда $d(W, \Pi) \rightarrow 0$.

Доказательство теоремы 2. Воспользуемся функциональным вариантом метода Чена-Стейна (см. [12], гл. 10). Введем множества $G_{l}(\alpha)$, $l=1, \ldots, M$, таких пар $\bar{j} \in J^{\left(s^{3}\right)}(k)$, для которых хотя бы один из отрезков

$$
\left[j_{1}-1, j_{1}+s+l\right],\left[j_{2}-1, j_{2}+s+l\right], \ldots,\left[j_{k}-1, j_{k}+s+l\right]
$$

лежит на расстоянии менее $s^{2}$ от какого-либо из отрезков

$$
\left[i_{1}-1, i_{1}+s+m\right],\left[i_{2}-1, i_{2}+s+m\right], \ldots,\left[i_{k}-1, i_{k}+s+m\right] .
$$

Каждой паре $\alpha=(\bar{i}, m) \in U$ сопоставим множество пар $\beta=(\bar{j}, l)$ из $U$

$$
\Gamma(\alpha)=\bigcup_{l=1}^{M} \Gamma_{l}(\alpha) \cup \bigcup_{l \neq m}\{(\bar{i}, l)\},
$$

где

$$
\Gamma_{l}(\alpha)=\left\{(\bar{j}, l): \bar{j} \in G_{l}(\alpha) \backslash\{\bar{i}\}\right\} .
$$

Отметим сразу, что при любом $\alpha \in U$

$$
\begin{gathered}
\left|G_{l}(\alpha)\right|<k C_{n}^{k-1}\left(s^{2}+s+l\right), \\
|\Gamma(\alpha)|=\sum_{l=1}^{M}\left|\Gamma_{l}(\alpha)\right|+M-1<M\left(k C_{n}^{k-1}\left(s^{2}+s+M\right)+1\right) .
\end{gathered}
$$

Пусть

$$
V_{\alpha}=\sum_{\beta=(\bar{i}, m) \in U \backslash(\Gamma(\alpha) \cup\{\alpha\})} \eta^{(l)}(\bar{j}) .
$$

Согласно теореме 10.А из [12]

$$
\begin{gathered}
d(W, \Pi) \leq \sum_{\alpha \in U}\left(\mathbf{E} \eta^{(m)}(\bar{i})\left(\mathbf{E} \eta^{(m)}(\bar{i})+\sum_{\beta \in \Gamma(\alpha)} \mathbf{E} \eta^{(l)}(\bar{j})\right)\right)+ \\
+\sum_{\alpha \in U} \sum_{\beta \in \Gamma(\alpha)} \mathbf{E}\left(\eta^{(m)}(\bar{i}) \eta^{(l)}(\bar{j})\right)+\sum_{\alpha \in U} \mathbf{E}\left|\mathbf{E} \eta^{(m)}(\bar{i})-\mathbf{E}\left(\eta^{(m)}(\bar{i}) \mid V_{\alpha}\right)\right| .
\end{gathered}
$$

Учитывая (20), (16) и условия теоремы, получаем

$$
\begin{gathered}
\sum_{\alpha \in U}\left(\mathbf{E} \eta^{(m)}(\bar{i})\left(\mathbf{E} \eta^{(m)}(\bar{i})+\sum_{\beta \in \Gamma(\alpha)} \mathbf{E} \eta^{(l)}(\bar{j})\right)\right)= \\
=O\left(\frac{s^{2}}{n}\left(\sum_{m=1}^{M} \mathbf{E} \delta_{n, k}^{(m)}(s)\right)^{2}\right)=o(1) .
\end{gathered}
$$


Далее, для любой пары $\alpha \in U$ разобьем каждое множество $G_{l}(\bar{i})$ на несколько подмножеств. Определим их свойствами входящих в них наборов $\bar{j}$ следующим образом:

$G_{l, a}(\alpha)$ - ровно $а$ отрезков $\left[j_{1}-1, j_{1}+s+l\right], \ldots,\left[j_{k}-1, j_{k}+s+l\right]$ лежат на расстоянии менее $s^{2}$ от одного из отрезков $\left[i_{1}-1, i_{1}+s+m\right], \ldots,\left[i_{k}-\right.$ $\left.-1, i_{k}+s+m\right]$, а остальные отрезки лежат на расстоянии не менее $s^{2}$ друг от друга, здесь $0 \leq a \leq k-1$,

$G_{l, k}^{\prime}(\alpha)-$ каждый из $k$ отрезков $\left[j_{1}-1, j_{1}+s+l\right], \ldots,\left[j_{k}-1, j_{k}+s+l\right]$ лежит на расстоянии менее $s^{2}$ от соответствующего отрезка среди $\left[i_{1}-1, i_{1}+\right.$ $+s+m], \ldots,\left[i_{k}-1, i_{k}+s+m\right]$, при этом хотя бы один не пересекается с этим отрезком более чем на одну позицию,

$$
\begin{aligned}
& G_{l, k}^{\prime \prime}(\alpha) \text { - для } \bar{j} \text { выполнена система соотношений } \\
& -l<j_{1}-i_{1}=\ldots=j_{k}-i_{k}<m, \\
& \begin{array}{c}
G_{l, k}^{\prime \prime \prime}(\alpha)=G_{l, k}(\alpha) \backslash\left(G_{l, k}^{\prime}(\alpha) \cup G_{l, k}^{\prime \prime}(\alpha)\right) . \\
\text { Аналогичным образом разобьем множества } \Gamma_{l}(\alpha): \\
\Gamma_{l, a}(\alpha)=G_{l, a}(\alpha) \times\{l\}, \quad \Gamma_{l, k}^{\prime}(\alpha)=G_{l, k}^{\prime}(\alpha) \times\{l\}, \\
\Gamma_{l, a}^{\prime \prime}(\alpha)=G_{l, a}^{\prime \prime}(\alpha) \times\{l\}, \quad \Gamma_{l, k}^{\prime \prime \prime}(\alpha)=G_{l, k}^{\prime \prime \prime}(\alpha) \times\{l\} .
\end{array}
\end{aligned}
$$

Из определений следует, что

$$
\begin{gathered}
\left|G_{l, a}(\alpha)\right|=O\left(s^{2 a} n^{2 k-a}\right), \quad 1 \leq a \leq k-1, \\
\left|G_{l, k}^{\prime}(\alpha)\right|=O\left(s^{2 k} n^{k}\right), \quad\left|\Gamma_{l, k}^{\prime \prime \prime}(\alpha)\right|=O\left(s^{2 k} n^{k}\right) .
\end{gathered}
$$

Кроме того,

$$
\begin{gathered}
\mathbf{P}\left\{\eta^{(l)}(\bar{j})=1 \mid \eta^{(m)}(\bar{i})=1\right\} \leq p^{(k-\alpha) s}, \quad \bar{j} \in \Gamma_{l, a}(\alpha), \\
\mathbf{P}\left\{\eta^{(l)}(\bar{j})=1 \mid \eta^{(m)}(\bar{i})=1\right\} \leq p^{2 s}, \quad \bar{j} \in \Gamma_{l, k}^{\prime}(\alpha), \\
\mathbf{P}\left\{\eta^{(l)}(\bar{j})=\eta^{(m)}(\bar{i})=1\right\}=0, \quad \bar{j} \in \Gamma_{l, k}^{\prime \prime}(\alpha), \quad l \neq m, \\
\quad \mathbf{P}\left\{\eta^{(l)}(\bar{j})=\eta^{(m)}(\bar{i})=1\right\} \leq p^{k s}, \quad \bar{j} \in \Gamma_{l, k}^{\prime \prime \prime}(\alpha) .
\end{gathered}
$$

Первые три оценки вытекают непосредственно из определений. Последняя оценка доказывается индукцией по $k \geq 2$.

По аналогии с рассуждениями в [2,9] и [11], используя приведенные выше оценки и (3), получаем оценки

$$
\sum_{\alpha \in U} \sum_{\beta \in \Gamma_{l, a}(\alpha)} \mathbf{E}\left(\eta^{(m)}(\bar{i}) \eta^{(l)}(\bar{j})\right)=
$$




$$
\begin{gathered}
=\sum_{\alpha \in U} \mathbf{P}\left\{\eta^{(m)}(\bar{i})=1\right\} \sum_{\beta \in \Gamma_{l, a}(\alpha)} \mathbf{P}\left\{\eta^{(l)}(\bar{j})=1 \mid \eta^{(m)}(\bar{i})=1\right\}= \\
=O\left(\left(\sum_{m=1}^{M} \mathbf{E} \delta_{n}^{(m)}(s)\right) s^{2 a}\left(n p^{s}\right)^{k-a}\right), \quad a=1, \ldots, k-1, \\
\sum_{\alpha \in U} \sum_{\beta \in \Gamma_{l, k}^{\prime}(\alpha)} \mathbf{E}\left(\eta^{(m)}(\bar{i}) \eta^{(l)}(\bar{j})\right)= \\
=\sum_{\alpha \in U} \mathbf{P}\left\{\eta^{(m)}(\bar{i})=1\right\} \sum_{\beta \in \Gamma_{l, k}^{\prime}(\alpha)} \mathbf{P}\left\{\eta^{(l)}(\bar{j})=1 \mid \eta^{(m)}(\bar{i})=1\right\}= \\
\left.=O\left(\sum_{m=1}^{M} \mathbf{E} \delta_{n}^{(m)}(s)\right) s^{2 k} p^{2 s}\right), \\
\sum_{\alpha \in U} \sum_{l \neq m} \sum_{\beta \in \Gamma_{l, a}^{\prime \prime}(\alpha)} \mathbf{E}\left(\eta^{(m)}(\bar{i}) \eta^{(l)}(\bar{j})\right)=0, \\
\mathbf{E}\left(\eta^{(m)}(\bar{i}) \eta^{(l)}(\bar{j})\right)=O\left(s^{2 k}\left(n p^{s}\right)^{k}\right) .
\end{gathered}
$$

Из этих оценок, формулы (19) и условий теоремы (напомним, что из них, в частности, вытекает соотношение $s=O(\ln n))$ следует, что

$$
\sum_{\alpha \in U} \sum_{\beta \in \Gamma(\alpha)} \mathbf{E}\left(\eta^{(m)}(\bar{i}) \eta^{(l)}(\bar{j})\right)=O\left(s^{2 k}\left(\left(n p^{s}\right)^{k}+p^{2 s}\right)\right) \rightarrow 0 .
$$

Далее оценим величину

$$
\sum_{\alpha \in U} \mathbf{E}\left|\mathbf{E} \eta^{(m)}(\bar{i})-\mathbf{E}\left(\eta^{(m)}(\bar{i}) \mid V_{\alpha}\right)\right| .
$$

Отметим, что область суммирования в определении величины $V_{\alpha}$ допускает оценку

$$
U \backslash(\Gamma(\alpha) \cup\{\alpha\}) \subseteq \bigcup_{l=1}^{M}\left(G_{l, 0}(\alpha) \times\{l\}\right) \subseteq G_{1,0}(\alpha) \times\{1, \ldots, M\}
$$

(множество $G_{l, 0}(\alpha)$ определено при выводе (23)). Значит, для каждого слагаемого в (24) выполнено неравенство

$$
\mathbf{E}\left|\mathbf{E} \eta^{(m)}(\bar{i})-\mathbf{E}\left(\eta^{(m)}(\bar{i}) \mid V_{\alpha}\right)\right| \leq \mathbf{E}\left|\mathbf{E} \eta^{(m)}(\bar{i})-\mathbf{E}\left(\eta^{(m)}(\bar{i}) \mid\left\{X_{t}, t \in T(\alpha)\right\}\right)\right|,
$$


где

$$
T(\alpha)=\bigcup_{u=1}^{k+1} T_{u}(\alpha), \quad T_{u}(\alpha)=\left\{i_{u-1}+s+s^{2}+1, \ldots, i_{u}-s^{2}-1\right\}
$$

(здесь и в следующих оценках считается, что $i_{0}+s+s^{2}=0$, a $i_{k+1}-s^{2}=n$ ). С учетом марковского свойства цепи $\mathbf{X}$ это приводит к неравенству

$$
\mathbf{E}\left|\mathbf{E} \eta^{(m)}(\bar{i})-\mathbf{E}\left(\eta^{(m)}(\bar{i}) \mid V_{\alpha}\right)\right| \leq \mathbf{E}\left|\mathbf{E} \eta^{(m)}(\bar{i})-\mathbf{E}\left(\eta^{(m)}(\bar{i}) \mid\left\{X_{t}, t \in T^{\prime}(\alpha)\right\}\right)\right|,
$$

где

$$
T^{\prime}(\alpha)=\bigcup_{u=1}^{k+1}\left\{i_{u-1}+s+s^{2}+1, i_{u}-s^{2}-1\right\}
$$

Формула (25) позволяет при оценке величины (24) почти дословно повторить рассуждения, использованные при выводе формулы (20) в [9]. Единственное существенное изменение касается числа оцениваемых разностей в разложении для правой части (25) (подробности см. в [9]). Теперь оцениваются $2^{k}$ разностей. Повторив упомянутые рассуждения, получим неравенство

$$
\begin{gathered}
\mathbf{E}\left|\mathbf{E} \eta^{(m)}(\bar{i})-\mathbf{E}\left(\eta^{(m)}(\bar{i}) \mid\left\{X_{t}, t \in T^{\prime}(\alpha)\right\}\right)\right| \leq \\
\leq 2^{k} \rho_{k}^{s-1}\left(1-\rho_{k}\right) e^{-c_{2}\left(s^{2}-2 s\right)} \sum_{r=1}^{N} \pi_{k, r}^{\prime} z_{k, r}^{-1} \sum_{l=1}^{N} \pi_{l}^{k} z_{k, l} .
\end{gathered}
$$

Поэтому из (3) и оценки $\left|J^{\left(s^{3}\right)}(k)\right|<n^{k}$ следует, что

$$
\sum_{\alpha \in U} \mathbf{E}\left|\mathbf{E} \eta^{(m)}(\bar{i})-\mathbf{E}\left(\eta^{(m)}(\bar{i}) \mid V_{\alpha}\right)\right|=O\left(\Lambda_{s, n}(k) e^{-c s^{2}}\right)
$$

при любом $0<c<c_{4}$.

Подставляя оценки (16), (22)-(26) в (21) и используя условие $s^{3}<n$, убеждаемся в том, что $d(W, \Pi) \rightarrow 0$. Теорема 2 доказана.

Этим доказательство утверждения 1) теоремы 1 завершено. 


\section{7. Доказательство утверждений 2) и 3) теоремы 1}

Воспользуемся вытекающими из определений формулами

$$
\tilde{\xi}_{n, k}(s)=\sum_{m \geq 1} \xi_{n, k}^{(m)}(s), \quad \xi_{n, k}(s)=\sum_{m \geq 1} m \xi_{n, k}^{(m)}(s) .
$$

Заметим, что

$$
\begin{gathered}
\mathbf{P}\left\{\tilde{\xi}_{n, k}(s) \neq \sum_{m=1}^{M} \xi_{n, k}^{(m)}(s)\right\} \leq \mathbf{P}\left\{\sum_{m \geq M+1} \xi_{n, k}^{(m)}(s) \geq 1\right\}, \\
\mathbf{P}\left\{\xi_{n, k}(s) \neq \sum_{m=1}^{M} m \xi_{n, k}^{(m)}(s)\right\} \leq \mathbf{P}\left\{\sum_{m \geq M+1} \xi_{n, k}^{(m)}(s) \geq 1\right\} .
\end{gathered}
$$

Оценим выражение в правых частях этих неравенств. Для этого воспользуемся неравенством

$$
\begin{gathered}
\mathbf{P}\left\{\sum_{m \geq M+1} \xi_{n, k}^{(m)}(s) \geq 1\right\} \leq \\
\leq \sum_{m \geq M+1} \mathbf{P}\left\{\xi_{n, k}^{(m)}(s) \neq \delta_{n, k}^{(m)}(s)\right\}+\sum_{m \geq M+1} \mathbf{P}\left\{\delta_{n, k}^{(m)}(s) \geq 1\right\},
\end{gathered}
$$

оценками (14) и (16) и условиями теоремы:

$$
\begin{gathered}
\sum_{m \geq M+1} \mathbf{P}\left\{\xi_{n, k}^{(m)}(s) \neq \delta_{n, k}^{(m)}(s)\right\}=O\left(\sum_{m \geq M+1}(s+m-1)^{3 k}\left(\frac{p}{\rho_{k}^{1 / k}}\right)^{s+m-1}\right)= \\
=O\left(\left(\frac{p(1+\varepsilon)}{\rho_{k}^{1 / k}}\right)^{s+M}\right) \forall \varepsilon>0, \\
\sum_{m \geq M+1} \mathbf{P}\left\{\delta_{n, k}^{(m)}(s) \geq 1\right\} \leq \sum_{m \geq M+1} \mathbf{E} \delta_{n, k}^{(m)}(s)= \\
\sum_{m \geq M+1} \Lambda_{s, n}(k) \rho_{k}^{m-1}\left(1-\rho_{k}\right)\left(1+O\left(\frac{s^{3}}{n}+e^{-c_{1} s}\right)\right)= \\
=O\left(\sum_{m \geq M+1} \rho_{k}^{m-1}\left(1-\rho_{k}\right)\right)=O\left(\rho_{k}^{M}\right) .
\end{gathered}
$$


Последние две оценки и (30) означают, что найдется такое число $\varepsilon>0$, удовлетворяющее неравенству $p(1+\varepsilon)<\rho_{k}^{1 / k}$, и такое число $C<\infty$, что

$$
\mathbf{P}\left\{\sum_{m \geq M+1} \xi_{n, k}^{(m)}(s) \geq 1\right\} \leq C\left(\left(\frac{p(1+\varepsilon)}{\rho_{k}^{1 / k}}\right)^{s+M}+\rho_{k}^{M}\right) .
$$

Рассуждения, аналогичные приведенным при выводе оценки (18), показывают, что при любом фиксированном $M \geq 1$

$$
\begin{gathered}
d\left(\mathcal{L}\left(\sum_{m=1}^{M} \xi_{n, k}^{(m)}(s)\right), \operatorname{Po}\left(\sum_{m=1}^{M} \mathbf{E} \delta_{n, k}^{(m)}(s)\right)\right) \leq d(W, \Pi), \\
d\left(\mathcal{L}\left(\sum_{m=1}^{M} m \xi_{n, k}^{(m)}(s)\right), \operatorname{Po}\left(\mathbf{E} \delta_{n, k}^{(1)}(s), \ldots, \mathbf{E} \delta_{n, k}^{(M)}(s)\right)\right) \leq d(W, \Pi) .
\end{gathered}
$$

Из (28), (29) и (31) следует, что выбором достаточно большого $M$ можно сделать вероятности

$$
\mathbf{P}\left\{\tilde{\xi}_{n, k}(s) \neq \sum_{m=1}^{M} \xi_{n, k}^{(m)}(s)\right\}, \quad \mathbf{P}\left\{\xi_{n, k}(s) \neq \sum_{m=1}^{M} m \xi_{n, k}^{(m)}(s)\right\}
$$

сколь угодно малыми. В свою очередь, из (17), (32), (33) и теоремы 2 следует, что при таком $M$ при переходе к пределу по $n, s \rightarrow \infty$

$$
\begin{gathered}
\mathcal{L}\left(\sum_{m=1}^{M} \xi_{n, k}^{(m)}(s)\right) \rightarrow \operatorname{Po}\left(\sum_{m=1}^{M} \lambda \rho_{k}^{m-1}\left(1-\rho_{k}\right)\right), \\
\mathcal{L}\left(\sum_{m=1}^{M} m \xi_{n, k}^{(m)}(s)\right) \rightarrow \operatorname{Po}\left(\lambda\left(1-\rho_{k}\right), \ldots, \lambda \rho_{k}^{M-1}\left(1-\rho_{k}\right)\right) .
\end{gathered}
$$

Наконец, выбором достаточно большого $M$ можно сделать величины

$$
\begin{gathered}
d\left(\operatorname{Po}\left(\sum_{m=1}^{M} \lambda \rho_{k}^{m-1}\left(1-\rho_{k}\right)\right), \operatorname{Po}\left(\sum_{m \geq 1} \lambda \rho_{k}^{m-1}\left(1-\rho_{k}\right)\right)\right), \\
d\left(\operatorname{Po}\left(\lambda\left(1-\rho_{k}\right), \ldots, \lambda \rho_{k}^{M-1}\left(1-\rho_{k}\right)\right), \operatorname{Po}\left(\lambda\left(1-\rho_{k}\right), \lambda \rho_{k}\left(1-\rho_{k}\right), \ldots\right)\right)
\end{gathered}
$$

сколь угодно малыми.

Из всего перечисленного следуют утверждения 2) и 3) теоремы 1, что проверяется стандартными рассуждениями.

Авторы признательны А. М. Зубкову и Андрею В. Иванову за полезные замечания. 


\section{Список литературы}

[1] Беляев П. Ф., “О совместном распределении частот длинных $s$-цепочек в мультиномиальной схеме с равновероятными исходами”, Теория вероятн. и примен., 14:3 (1969), 540-546.

[2] Зубков А.М., Михайлов В.Г., “Предельные распределения случайных величин, связанных с длинными повторениями в последовательности независимых испытаний”, Теория вероятн. и примен., 19:1 (1974), 173-181.

[3] Зубков А. М., Михайлов В. Г., “О повторениях $s$-цепочек в последовательности независимых величин”, Теория вероятн. и примен., 24:2 (1979), 267-279.

[4] Михайлов В.Г., “Предельные распределения случайных величин, связанных с многократными длинными повторениями в последовательности независимых испытаний”, Теория вероятн. и примен., 19:1 (1974), 182-187.

[5] Hansen N. R., "Local alignment of Markov chains", Ann. Appl. Probab., 16:3 (2006), $1262-1296$.

[6] Беляев П. Ф., “К вопросу о совместном распределении частот $s$-цепочек в сложных цепях Маркова с большим числом состояний”, Теория вероятн. и примен., 14:2 (1969), 333-339.

[7] Беляев П.Ф. О совместном распределении частот исходов в цепях Маркова с большим числом состояний, Теория вероятн. и примен., 22:3 (1977), 534-545.

[8] Зубков А. М., "Неравенства для вероятностей переходов с запрещениями и их применения”, Матем. сб., 109(151):4(8) (1979), 491-532.

[9] Михайлов В.Г., Шойтов А.М., “О длинных повторениях цепочек в цепи Маркова”, Дискретная математика, 26:3 (2014), 79-89.

[10] Гантмахер Ф. Р., Теория матрии, 5-е изд., М.: ФИЗМАТЛИТ, 2004, 560 с.

[11] Шойтов А.М., "Пуассоновское приближение для числа повторений значений дискретной функции от цепочек”, Дискретная математика, 17:2 (2005), 56-69.

[12] Barbour A.D., Holst L., Janson S., Poisson Approximation, Oxford: Oxford University Press, 1992, 277 pp. 